\title{
Trends in incidence and medical resource utilisation in patients with chronic lymphocytic leukaemia: insights from the UK Clinical Practice Research Datalink (CPRD)
}

\author{
A. M. Pfeil • P. Imfeld • R. Pettengell • S. S. Jick • \\ T. D. Szucs • Christoph R. Meier • M. Schwenkglenks
}

Received: 27 June 2014 / Accepted: 6 September 2014 / Published online: 16 September 2014

(C) Springer-Verlag Berlin Heidelberg 2014

\begin{abstract}
Chronic lymphocytic leukaemia (CLL) is the most common leukaemia in European adults. We aimed to evaluate time trends in CLL incidence and medical resource utilisation of CLL patients in the UK. We conducted a retrospective, observational cohort analysis using the UK Clinical Practice Research Datalink (CPRD) comprising mainly primary care data. We included adult patients with newly diagnosed CLL between January 2000 and June 2012. Descriptive and trend analyses of CLL incidence and medical resource utilisation were performed. A total of 2576 patients with CLL met the eligibility criteria. At diagnosis, the majority of patients (71.7\%) were above 65 years of age. The European agestandardised CLL incidence rate in the CPRD was 6.2/ 100,000 (95\% confidence interval [CI] 6.0, 6.5/100,000) person-years. There was no statistically significant increase over time. The CLL patients had on average 74.6 general practitioner visits during a median follow-up of 3.3 years. Between 2000 and 2012, the average number of recorded
\end{abstract}

\author{
A. M. Pfeil • T. D. Szucs $\cdot$ M. Schwenkglenks \\ Institute of Pharmaceutical Medicine (ECPM), University of Basel, \\ Basel, Switzerland \\ P. Imfeld $\cdot$ C. R. Meier $(\bowtie)$ \\ Basel Pharmacoepidemiology Unit, Division of Clinical Pharmacy \\ and Epidemiology, Department of Pharmaceutical Sciences, \\ University of Basel, Spitalstrasse 26, 4031 Basel, Switzerland \\ e-mail: Christoph.Meier@usb.ch \\ P. Imfeld • C. R. Meier \\ Hospital Pharmacy, University Hospital Basel, Spitalstrasse 26, \\ 4031 Basel, Switzerland \\ R. Pettengell \\ Cellular and Molecular Medicine, St. George's University of \\ London, London, UK \\ S. S. Jick $\cdot$ C. R. Meier \\ Boston Collaborative Drug Surveillance Program, Boston University \\ School of Public Health, Lexington, MA, USA
}

hospitalisations and referrals per year corrected for duration of follow-up significantly $(p<0.001)$ increased by $8.1 \%$ (95\% CI $6.8 \%, 9.3 \%)$ and $16.4 \%$ (95\% CI $15.4 \%$, $17.3 \%$ ), respectively. Referrals and hospitalisations in the second year compared to the first year following the CLL diagnosis significantly decreased. CLL incidence rates in the CPRD were stable over the period from 2000 to 2012. Medical resource utilisation in UK primary care was well documented, but further research is needed to describe secondary and tertiary care medical resource utilisation e.g. chemotherapy administration, which is inadequately captured in the CPRD.

Keywords Incidence $\cdot$ Chronic lymphocytic leukaemia · Epidemiology $\cdot$ Mortality $\cdot$ Resource utilisation $\cdot$ Cohort study

\section{Introduction}

Chronic lymphocytic leukaemia (CLL) is the most common leukaemia in adults. The clinical manifestations of CLL range from an asymptomatic presentation with minimal B cell lymphocytosis to a progressive clinical picture of enlarged lymph nodes, splenomegaly, anaemia and thrombocytopenia [1]. Median age at diagnosis has been reported to be 72 years [2], and males are more likely to develop CLL than females. Several prognostic markers such as age, stage, performance status, lymphocyte count, serum parameters and chromosomal abnormalities have been identified [1]. Survival time of patients with CLL ranges from less than 2 to 15 years or more with a median survival of about 10 years [3]. In the USA, CLL occurs with an estimated incidence of 3-5/100,000 people per year [2]. A crude incidence rate of 4.2/100,000 person-years has been reported for the UK in 2009 [4]. There are no current age-standardised incidence data for Europe. 
About $70 \%$ of patients with CLL are asymptomatic and at an early disease stage at the time of diagnosis [3]. Chemotherapy in CLL patients is not recommended until patients develop symptoms, have organ compromise or until the disease is rapidly progressing. Before initiating chemotherapy treatment, it is important to assess the fitness level of a CLL patient $[5,6]$. Depending on life expectancy, general health status and expected ability to tolerate aggressive chemotherapy, different chemotherapy regimens are used to treat CLL. Combination chemotherapy with fludarabine, cyclophosphamide and rituximab is considered as a standard for fit, chemotherapy-naive patients [7] but may not always be suitable for elderly patients or those with comorbidities. Chlorambucil in combination with an anti-CD20 monoclonal antibody such as rituximab or obinutuzumab is considered standard for elderly or comorbid patients [8].

As a result, various management strategies are possible. Intensive immuno-chemotherapy is given to fit patients with no or mild comorbidities and normal life expectancy (Go-Go) aiming at achieving prolonged progression-free and overall survival. Symptom relief and response to treatment are the main goals in the intermediate chemotherapy intensity group with moderate comorbidities and compromised life expectancy (Slow-Go). In patients with several or severe comorbidities and very short life expectancy, symptom management and prolongation of functional independence (No-Go) are the main objectives [9]. Instead of starting with the least aggressive chemotherapy, followed if needed by a second, more aggressive treatment regimen, the optimal chemotherapy is given to patients upfront to keep them in remission for as long as possible. The change in management of CLL patients from palliative to curative care and towards an individually tailored chemotherapy $[10,11]$, together with its impact on resource utilisation, is poorly studied.

We aimed to assess CLL incidence rates and to evaluate time trends in CLL incidence and medical resource utilisation of CLL patients using a primary care database from the UK.

\section{Methods}

Study design and data source

We conducted a retrospective, observational cohort study using the large, anonymised UK-based Clinical Practice Research Datalink (CPRD). The CPRD, formerly known as the General Practice Research Database (GPRD), established in 1987, is part of the UK National Health Service's (NHS) National Institute for Health Research (NIHR) and the Medicines and Healthcare Products Regulatory Agency (MHRA). The database contains primary care data on about $13 \%$ of the UK population and is representative of this population with respect to age, gender, regional distribution and annual turnover rate. General practitioners (GPs) are trained to record clinical data in a standardised manner. The MHRA anonymises the raw data before release and performs quality controls to ensure that the standards of the data collection are fulfilled. The database contains information on patient demographics such as age and gender, body mass index (BMI), region of residence, medical diagnoses, laboratory test results, information about referrals to specialists, hospitalisations and drug prescriptions.

This study was approved by the Independent Scientific Advisory Committee (ISAC) for MHRA database. Numerous studies involving cancer have been conducted using the CPRD [12-14], and the validity of the data recorded in the database has been reported to be high [15].

\section{Study population}

The study population consisted of patients with a diagnosis of CLL between January 2000 and June 2012 aged 18 years or older at the time of diagnosis. Subjects with less than 3 years of recorded history prior to the CLL diagnosis were excluded in order to increase the likelihood of capturing truly incident cases. Patients diagnosed with CLL before 2000 were excluded because the degree of consistency of the recording of the CLL diagnosis and the medical resource utilisation in the CPRD before 2000 was unclear. We validated the CLL diagnosis by screening 100 randomly selected CLL patient records for typical diagnostic procedures such as blood tests, chest X-rays, bone marrow examinations and ultrasound scans; for symptoms such as swollen lymph glands, fever, unusual sweating and tiredness; for referrals to a specialist or specialised clinic; and for prescriptions such as chlorambucil, fludarabine, cyclophosphamide and rituximab. The CLL diagnosis was considered validated if the 100 CLL patient profiles included at least one screening criterion for CLL.

Follow-up and incidence rates

We followed all patients in the study population from the date of CLL diagnosis until they died, left the practice or reached the end of the study period. Incidence rates were calculated as the number of new cases divided by the total number of person-years at risk. We summarised person-years by year for all subjects enrolled in the CPRD with at least 3 years of prior history and at risk of CLL between 2000 and the end of follow-up (i.e. a CLL diagnosis, death, leaving the practice or the end of the study period). Age-standardised incidence rates were calculated based on the most recently updated European standard population [16]. 
Basic characteristics and Charlson comorbidity weighted index

For all patients in the study population, we assessed basic characteristics such as age at CLL diagnosis, gender, smoking status, alcohol consumption, BMI, body surface area (BSA), height and weight. For smoking status, alcohol consumption, BMI, BSA, height and weight, the values recorded closest and prior to the CLL diagnosis date were extracted. Additionally, we extracted all comorbidities included in the Charlson comorbidity weighted index, because they were associated with the risk of mortality [17].

Medical resource utilisation

We assessed medical resource utilisation between the CLL diagnosis and the end of follow-up, including number of GP visits, hospitalisations, referrals to specialists (such as oncologists, radiotherapists or haematologists) or specialised clinics, number of cancer-related therapies (such as chemotherapies or radiotherapies), blood transfusions, blood tests (such as red blood cell counts or neutrophil counts) and prescriptions for chemotherapeutics, antidepressants, antiemetics, antifungals, antibiotics, antivirals, immunosuppressants and growth factors.

\section{Descriptive and statistical analysis}

We summarised categorical data using standard descriptive statistics, i.e. number of observations, frequencies and percentages. Continuous data were reported using the mean and standard deviation (SD) if normally distributed. Median and interquartile range (IQR) or range were reported if the distribution was skewed. We performed log-linear Poisson regression to describe the change in age-adjusted incidence rate over time [18]. The age-specific incidence rates, expressed as the division of incident counts and corresponding person-years, were log-transformed. Person-years on the log scale were then added up and used in the model as an offset, a value that is known and not predicted by the regression. The offset was subsequently used to re-calculate the age-adjusted incidence rate from the estimated count.

We performed multivariable Cox regression [19] to determine the influence of age at diagnosis, gender, Charlson comorbidity weighted index and year of diagnosis on overall survival. Analyses of time trends in medical resource utilisation were performed using Poisson regression for count data. To correct for a possible cohort effect in the CPRD trend analyses of medical resource utilisation, years of follow-up were included in the Poisson regression model as an explanatory variable.

We carried out two-sided statistical tests at a $5 \%$ significance level, and $95 \%$ confidence intervals (CIs) were obtained, if applicable. All analyses were performed using Stata/SE version 12.1 (StataCorp LP, College Station, TX, USA).

\section{Results}

We identified $5266(0.05 \%)$ patients with a diagnosis of CLL from among around nine million subjects currently included in the CPRD. Of these, 2690 patients were excluded because they did not meet the eligibility criteria. Figure 1 shows details of the patient selection. We included 2576 patients with a diagnosis of CLL aged 18 years or older in the analyses, of whom 1498 (58.2\%) were male and 1078 (41.8\%) were female. Mean age at diagnosis was 70.9 years $(\mathrm{SD} \pm 11.3$ years, range $32-102$ years); a majority of patients ( $n=1847,71.7 \%)$ were of age 65 years or over. On average, CLL patients had 12.8 (SD 4.9)years of recorded prior history. Further characteristics of the study population are presented in Table 1.

\section{Incidence rates}

The overall crude CLL incidence rate was 7.2/100,000 (95\% CI $6.9,7.4 / 100,000)$ person-years, and the overall agestandardised incidence rate was 6.2/100,000 (95\% CI 6.0, $6.5 / 100,000)$ person-years. Age-standardised incidence rates in 2000 and 2010 were 5.6/100,000 (95\% CI 4.7, 6.4/ $100,000)$ person-years and 6.4/100,000 (95 \% CI 5.5, 7.2/ 100,000)person-years, respectively (Fig. 2). This corresponds to an average annual increase of $0.2 \%$, which did not reach statistical significance $(p=0.787)$. In some years, rates of up to 6.8/100,000 (95 \% CI 5.9, 7.7/100,000)person-years were seen. There was a decline in the age-standardised incidence rate in 2012. In the past 5 years, we observed a stabilisation of CLL incidence rates in the CPRD population.

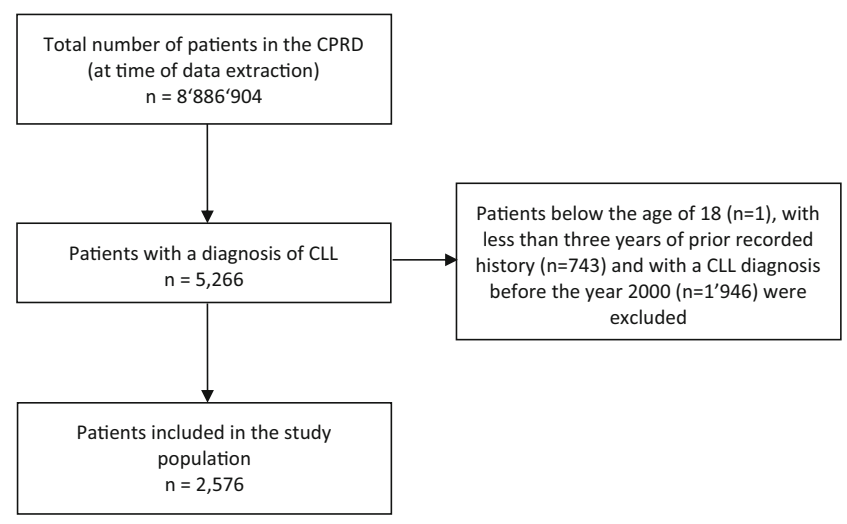

Fig. 1 Patient selection flow diagram 
Table 1 Characteristics of the study population

\begin{tabular}{|c|c|c|c|}
\hline & \multicolumn{3}{|c|}{ Patients } \\
\hline & \multicolumn{2}{|c|}{$N=2576$} & $\%$ \\
\hline Age at diagnosis & \multicolumn{2}{|l|}{2576} & 100.0 \\
\hline $18-32$ years & \multicolumn{2}{|l|}{0} & 0.0 \\
\hline $32-62$ years & \multicolumn{2}{|l|}{607} & 23.6 \\
\hline $63-71$ years & \multicolumn{2}{|l|}{672} & 26.1 \\
\hline $72-78$ years & \multicolumn{2}{|l|}{598} & 23.2 \\
\hline $79+$ years & \multicolumn{2}{|l|}{699} & 27.1 \\
\hline Gender & \multicolumn{2}{|l|}{2576} & 100.0 \\
\hline Female & \multicolumn{2}{|l|}{1078} & 41.8 \\
\hline Male & \multicolumn{2}{|l|}{1498} & 58.2 \\
\hline Smoking status & \multicolumn{2}{|l|}{2576} & 100.0 \\
\hline Non-smoker & \multicolumn{2}{|l|}{1156} & 44.9 \\
\hline Current smoker & \multicolumn{2}{|l|}{252} & 9.8 \\
\hline Former smoker & \multicolumn{2}{|l|}{1053} & 44.0 \\
\hline Unknown & \multicolumn{2}{|l|}{33} & 1.3 \\
\hline Alcohol consumption & \multicolumn{2}{|l|}{2576} & 100.0 \\
\hline Never & \multicolumn{2}{|l|}{439} & 17.0 \\
\hline Current & \multicolumn{2}{|l|}{1846} & 71.7 \\
\hline Former & \multicolumn{2}{|l|}{65} & 2.5 \\
\hline Unknown & \multicolumn{2}{|l|}{226} & 8.8 \\
\hline Body mass index $\left(\mathrm{kg} / \mathrm{m}^{2}\right) 26.6 \pm 4.8^{\mathrm{a}}$ & \multicolumn{2}{|l|}{2388} & 92.7 \\
\hline Body surface area $\left(\mathrm{m}^{2}\right) 1.9 \pm 0.2^{\mathrm{a}}$ & 2384 & & 92.5 \\
\hline & $n$ & Men $(\%)$ & Women $(\%)$ \\
\hline Comorbidities & & & \\
\hline None & 1529 & $873(33.9)$ & $656(25.5)$ \\
\hline COPD & 189 & $136(5.3)$ & $53(2.0)$ \\
\hline Congestive heart failure & 95 & $64(2.5)$ & $31(1.2)$ \\
\hline Myocardial infarction & 65 & $46(1.8)$ & $19(0.7)$ \\
\hline Hypertension & 406 & $221(8.6)$ & $185(7.2)$ \\
\hline Hypotension & 55 & $37(1.4)$ & $18(0.7)$ \\
\hline Diabetes & 215 & $127(4.9)$ & $88(3.4)$ \\
\hline Peripheral vascular disease & 82 & $53(2.1)$ & $29(1.1)$ \\
\hline Cerebrovascular disease & 73 & $44(1.7)$ & $29(1.1)$ \\
\hline Renal diseases & 392 & $209(8.1)$ & $183(7.1)$ \\
\hline Ulcer & 187 & $105(4.1)$ & $82(3.2)$ \\
\hline Liver disease & 8 & $8(0.3)$ & $0(0.0)$ \\
\hline Connective tissue disease & 3 & $3(0.1)$ & $0(0.0)$ \\
\hline Hemiplegia & 2 & $2(0.0)$ & $0(0.0)$ \\
\hline Dementia & 60 & $28(1.1)$ & $32(1.2)$ \\
\hline All tumours & 7 & $5(0.2)$ & $2(0.0)$ \\
\hline Metastatic solid tumour & 7 & $5(0.2)$ & $2(0.0)$ \\
\hline Lymphoma $^{\mathrm{b}}$ & 86 & $53(2.1)$ & $33(1.3)$ \\
\hline Secondary malignancies ${ }^{\mathrm{c}}$ & 127 & $84(3.3)$ & $43(1.7)$ \\
\hline Charlson comorbidity weighted inde & & & \\
\hline 0 & 1529 & $873(33.9)$ & $656(25.5)$ \\
\hline 1 & 424 & $255(9.9)$ & $169(6.6)$ \\
\hline 2 & 376 & $220(8.5)$ & $156(6.1)$ \\
\hline 3 & 151 & $91(3.5)$ & $60(2.3)$ \\
\hline 4 & 52 & $31(1.2)$ & $21(0.8)$ \\
\hline
\end{tabular}

Table 1 (continued)

\begin{tabular}{rrrr}
\hline 5 & 18 & $12(0.5)$ & $6(0.2)$ \\
6 & 15 & $8(0.3)$ & $7(0.3)$ \\
7 & 4 & $3(0.1)$ & $1(0.0)$ \\
8 & 5 & $4(0.2)$ & $1(0.0)$ \\
9 & 2 & $1(0.0)$ & $1(0.0)$ \\
\hline
\end{tabular}

$\%$ given as the percentage of the total number of patients $(n=2576)$

$C O P D$ chronic obstructive pulmonary disease, $n$ number of patients, NOS not otherwise specified

${ }^{\mathrm{a}}$ Mean \pm standard deviation

${ }^{\mathrm{b}}$ Including patients with lymphoma NOS, non-Hodgkin lymphoma NOS and one patient with diffuse large B cell lymphoma

${ }^{\mathrm{c}}$ Other malignancies that occurred after the diagnosis of chronic lymphocytic leukaemia

Charlson comorbidity weighted index

During follow-up, the majority of patients ( $n=1425,60.1 \%$ ) in the study population had none of the comorbidities defined in the Charlson comorbidity weighted index (Table 1). Of those with comorbidities, 857 (90.5\%) had a Charlson comorbidity weighted index ranging from one to three. Chronic obstructive pulmonary disease $(n=189)$ was more common in men $(n=136)$ than in women $(n=53)(72$ vs. $28 \%, p<0.001)$, as were congestive heart failure ( $n=95,64$ men, 31 women, 67 vs. $33 \%, p=0.064)$ and hypertension $(n=406,221 \mathrm{men}$, 185 women, 54 vs. $46 \%, p=0.098)$, whereas dementia $(n=60$, 28 men, 32 women) was more common in women (53 vs. $47 \%, p=0.068$ ). Between 2000 and 2012, there was a significant trend of decreasing average Charlson comorbidity weighted index $(-7.4 \%, 95 \% \mathrm{CI}-9.1,-5.8 \%, p<0.001)$ per year.

Follow-up and survival

Median follow-up after the diagnosis of CLL was 3.3 years (IQR 4.7 years, range $0.02-12.4$ years). About a third of all CLL patients $(n=802,31.1 \%)$ died from any cause during follow-up. The overall Kaplan-Meier estimate of median survival was 8.6 years from the year of diagnosis (Fig. 3). Survival significantly $(p<0.001)$ differed by gender, age at diagnosis and Charlson comorbidity weighted index (Fig. 3): males had a shorter survival than females (adjusted hazard ratio [HR] 1.5, $95 \%$ CI 1.3, 1.7); survival decreased per year increase in age at diagnosis (adjusted HR 1.08, 95 \% CI 1.07, 1.09) and decreased with one unit increase in Charlson comorbidity weighted index (adjusted HR 2.3, $95 \%$ CI 1.5, 3.6). A CLL diagnosis in the years 2001 to 2012 compared to a CLL diagnosis in 2000 was borderline significantly associated $(p=0.077)$ with improved survival (HR 0.98, $95 \%$ CI $0.95,1.0)$. 


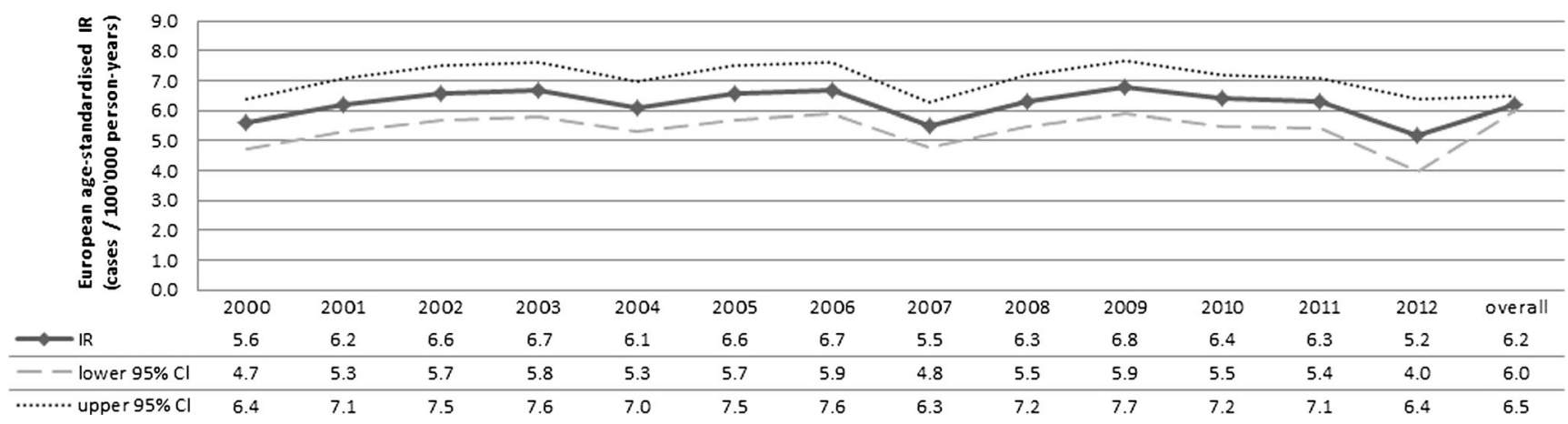

Fig. 2 Age-standardised incidence rates of CLL between 2000 and 2012. IR incidence rate, CI confidence interval

\section{Medical resource utilisation}

During a median follow-up of 3.3 (IQR 4.7)years after the diagnosis of CLL, patients had on average 74.6 GP visits, 3.2 referrals to specialists or specialised clinics, 0.5 hospitalisations and 0.1 chemotherapies or radiotherapies, as presented in detail in Table 2. Prescriptions of immunosuppressants, chemotherapeutics or antiemetics per patient per year were 0.1 prescriptions for immunosuppressants, 0.6 prescriptions for chemotherapeutics and 1.1 prescriptions for antiemetics, respectively. Prescriptions included chemotherapeutic agents such as fludarabine $(n=2)$, cyclophosphamide $(n=3)$, prednisolone a

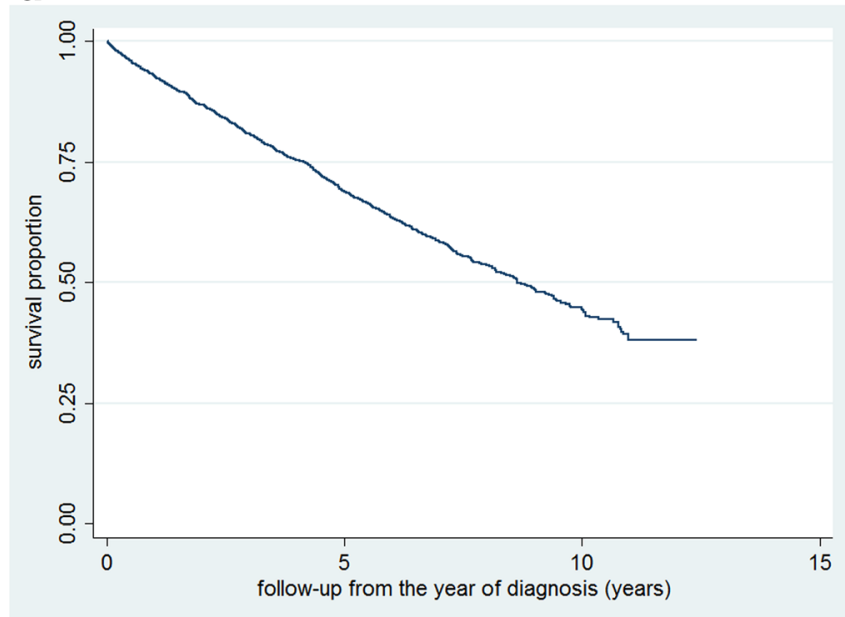

C

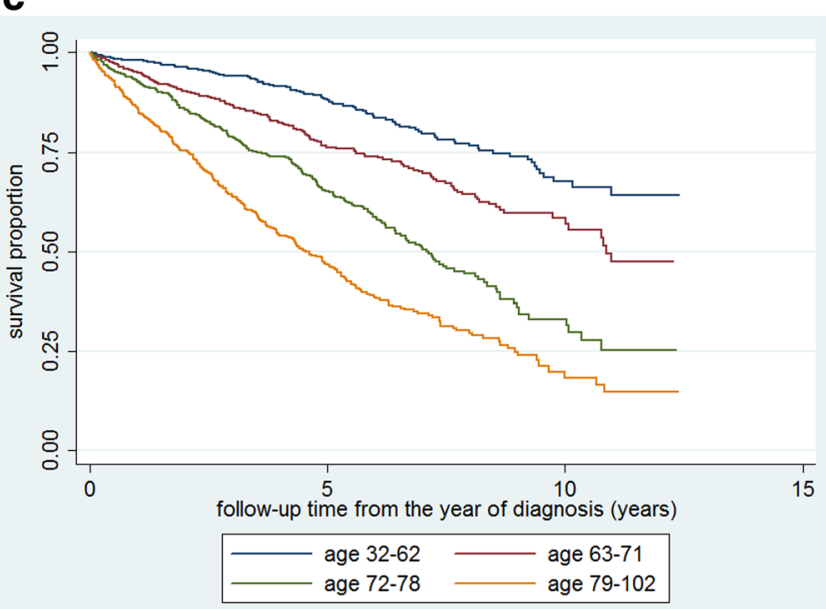

b

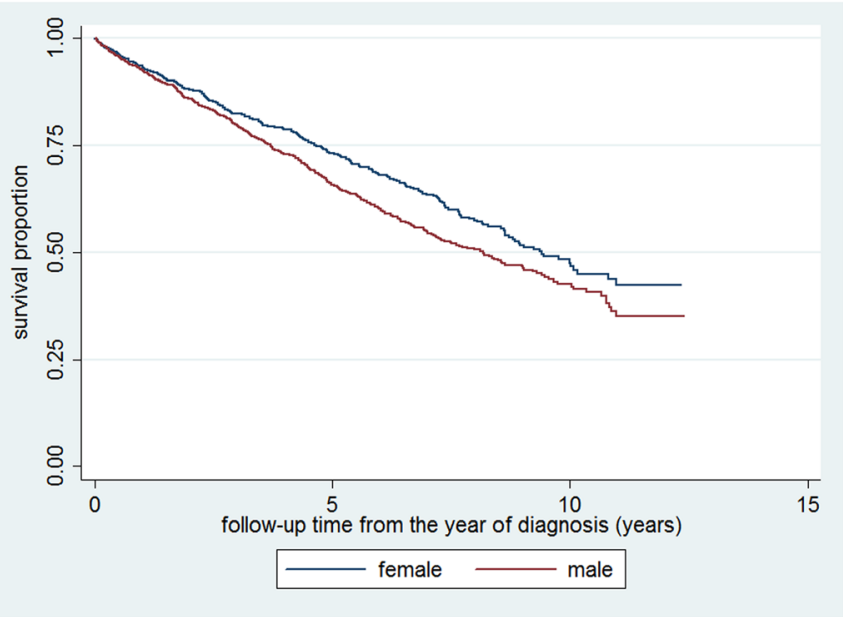

d

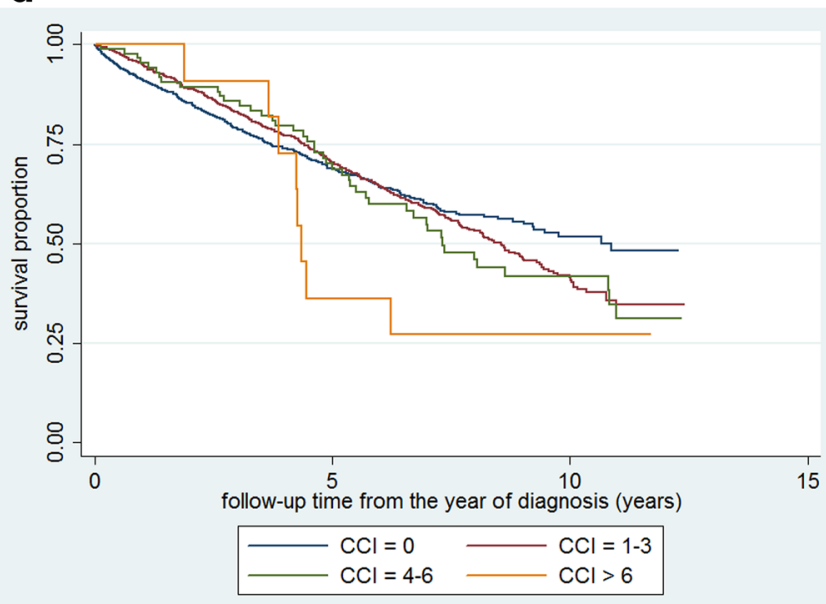

Fig. 3 Kaplan-Meier estimate of overall survival. Kaplan-Meier estimate of a overall survival, b overall survival stratified by gender, $\mathbf{c}$ overall survival stratified by age group and $\mathbf{d}$ overall survival stratified by Charlson comorbidity weighted index. CCI Charlson comorbidity weighted index 
Table 2 Medical resource utilisation from 2000 to 2012

\begin{tabular}{|c|c|c|c|c|c|c|}
\hline Item & $\begin{array}{l}\text { Average number during } \\
\text { mean follow-up } \\
\text { of } 4.1 \text { years }\end{array}$ & SD & Range & $\begin{array}{l}\text { Average number } \\
\text { per patient } \\
\text { per year }^{\mathrm{a}}\end{array}$ & SD & Range \\
\hline GP visits & 74.6 & 65.3 & $1-417$ & 22.2 & 17.1 & $1-302$ \\
\hline First visit after CLL diagnosis on average within (days) & 16.5 & 39.3 & $1-1304$ & NA & NA & NA \\
\hline In the first year following the CLL diagnosis & NA & NA & NA & 17.6 & 13.0 & $1-162$ \\
\hline Referrals & 3.2 & 4.4 & $0-65$ & 1.0 & 2.4 & $0-41$ \\
\hline $\begin{array}{l}\text { First referral after CLL diagnosis on average } \\
\text { within (years) }\end{array}$ & 1.1 & 1.5 & $0-9.6$ & NA & NA & NA \\
\hline In the first year following the CLL diagnosis & NA & NA & NA & 0.8 & 1.3 & $0-20$ \\
\hline Hospitalisations & 0.5 & 1.2 & $0-14$ & 0.3 & 2.2 & $0-81$ \\
\hline $\begin{array}{l}\text { First hospitalisation after CLL diagnosis on } \\
\text { average within (years) }\end{array}$ & 2.5 & 2.4 & $0-11.1$ & NA & NA & NA \\
\hline In the first year following the CLL diagnosis & NA & NA & NA & 0.1 & 0.5 & $0-7$ \\
\hline Chemotherapy and radiotherapy & 0.1 & 0.6 & $0-9$ & 0.05 & 0.3 & $0-12$ \\
\hline $\begin{array}{l}\text { First therapy after CLL diagnosis on average } \\
\text { within (years) }\end{array}$ & 2.5 & 2.4 & $0-10.4$ & NA & NA & NA \\
\hline Blood transfusion & 0.1 & 1.7 & $0-72$ & 0.06 & 0.7 & $0-21$ \\
\hline $\begin{array}{l}\text { First blood transfusion after CLL diagnosis } \\
\text { on average within (years) }\end{array}$ & 2.9 & 2.8 & $0-9.9$ & NA & NA & NA \\
\hline \multicolumn{7}{|l|}{ Tests and investigations } \\
\hline Bone marrow examination $(n=4)$ & 1.3 & 0.5 & $1-2$ & - & - & - \\
\hline Haemoglobin $(n=2146)$ & 8.2 & 10.0 & $1-136$ & 2.3 & 2.7 & $0.1-37$ \\
\hline Neutrophil counts $(n=2034)$ & 7.5 & 8.8 & $1-112$ & 2.1 & 2.6 & $0.1-37$ \\
\hline Red blood cell counts $(n=1953)$ & 6.4 & 7.7 & $1-95$ & 1.8 & 2.7 & $0.1-61$ \\
\hline White blood cell counts $(n=2137)$ & 8.2 & 10.0 & $1-138$ & 2.3 & 2.8 & $0.1-39$ \\
\hline Platelets $(n=2131)$ & 9.0 & 11.2 & $1-138$ & 2.5 & 3.2 & $0.1-61$ \\
\hline \multicolumn{7}{|l|}{ Prescriptions } \\
\hline Chemotherapeutics & 1.9 & 14.3 & $0-595$ & 0.6 & 2.9 & $0-69$ \\
\hline Immunosuppresives & 0.4 & 6.8 & $0-297$ & 0.1 & 1.1 & $0-35$ \\
\hline Antiemetics & 3.6 & 16.3 & $0-362$ & 1.1 & 4.3 & $0-101$ \\
\hline Antidepressants & 5.9 & 19.4 & $0-315$ & 1.6 & 4.8 & $0-56$ \\
\hline Antifungals & 0.7 & 2.9 & $0-55$ & 0.2 & 1.2 & $0-20$ \\
\hline Antibiotics & 7.0 & 12.7 & $0-158$ & 1.8 & 3.1 & $0-55$ \\
\hline Antivirals & 0.6 & 3.0 & $0-63$ & 0.1 & 0.8 & $0-16$ \\
\hline Growth factors & 0.03 & 0.8 & $0-35$ & 0.01 & 0.3 & $0-12$ \\
\hline \multicolumn{7}{|l|}{ Trend analyses } \\
\hline \multicolumn{4}{|l|}{ Item } & Estimate & $95 \%$ CI & $p$ value \\
\hline \multicolumn{4}{|l|}{ Change in average number of referrals per year } & $16.4 \%$ & $15.4 \%, 17.3 \%$ & $p<0.001$ \\
\hline \multicolumn{4}{|l|}{ Change in number of referrals per patient per year } & $0.05 \%$ & $0.04 \%, 0.06 \%$ & $p<0.001$ \\
\hline \multicolumn{4}{|c|}{$\begin{array}{l}\text { Change in average number of referrals in the second year compared to the first year following } \\
\text { the CLL diagnosis }\end{array}$} & $-35.3 \%$ & $-28.8 \%,-70.2 \%$ & $p<0.001$ \\
\hline \multicolumn{4}{|c|}{ Change in average number of hospitalisations per year } & $8.1 \%$ & $6.8 \%, 9.3 \%$ & $p<0.001$ \\
\hline \multicolumn{4}{|l|}{ Change in number of hospitalisations per patient per year } & $0.05 \%$ & $0.02 \%, 0.08 \%$ & $p=0.002$ \\
\hline \multicolumn{4}{|c|}{$\begin{array}{l}\text { Change in average number of hospitalisations in the second year compared to the first year } \\
\text { following the CLL diagnosis }\end{array}$} & $-29.4 \%$ & $-12.8 \%,-45.9 \%$ & $p<0.001$ \\
\hline
\end{tabular}

Where $n$ is not specified, the number was obtained including all patients

$C I$ confidence interval, $C L L$ chronic lymphocytic leukaemia, GP general practitioner, $N A$ not applicable, $S D$ standard deviation

${ }^{\mathrm{a}}$ Corrected for follow-up

$(n=709)$ and chlorambucil $(n=35)$. No prescription codes for monoclonal antibodies such as rituximab were recorded in our study population.
Between 2000 and 2012, the average number of recorded referrals to a specialist or specialised clinic and the average number of recorded hospitalisations per year (corrected for 
duration of follow-up) significantly increased by $8.1 \%(95 \%$ CI 6.8, $9.3 \%$ ) and $16.4 \%$ (95\% CI 15.4, $17.3 \%$ ), respectively. In the same time period, a small increase in the number of referrals and hospitalisations per patient per year (corrected for duration of follow-up) was observed (Table 2).

In the first year following the CLL diagnosis, 0.8 referrals were recorded on average. The average number of recorded referrals was 0.5 in the second year following the CLL diagnosis, corresponding to a significant decrease of $35.3 \%(95 \%$ CI 28.8, $70.2 \%$ ). On average, 0.13 hospitalisations were recorded in the first year following the CLL diagnosis and 0.09 hospitalisations in the second year following the CLL diagnosis. This corresponds to a significant decrease by $29.4 \%$ (95 \% CI 12.8, $45.9 \%)$.

\section{Discussion}

In this large epidemiological study based on primary care data from the UK CPRD, we identified 2576 eligible adult patients with a diagnosis of CLL between 2000 and 2012. The overall age-standardised incidence rate was 6.2/100,000 (95\% CI 6.0, $6.5 / 100,000)$ person-years. We found that CLL incidence rates in the UK CPRD increased slightly between 2000 and 2005 and then stabilised between 2006 and 2010. Male and elderly patients were more often diagnosed with CLL. More than a third of the patients had comorbidities defined in the Charlson comorbidity weighted index. Overall median survival after the CLL diagnosis was about 9 years and significantly differed by age, gender and Charlson comorbidity weighted index. The average number of recorded referrals to a specialist or specialised clinic and hospitalisations per patient per year significantly increased between 2000 and 2012. Referrals and hospitalisations were less frequent in the second year following the CLL diagnosis compared to the first year following the CLL diagnosis.

Our estimate of the crude incidence rate of CLL in the CPRD population (7.2/100,000, $95 \%$ CI 6.9, 7.4/100,000 person-years) was higher than the one reported in a Czech study (5.8 and 6.2/100,000 people in 2006 and 2007, respectively) [20]. Compared to our age-standardised incidence rate estimate of 6.2/100,000 person-years, lower cancer registry-based age-standardised incidence rates of CLL (3.8/100,000 personyears over a 20-year time period) were reported for the Netherlands [21] and for the UK (3.6/100,000 people in 2009) [22]. Our estimate was higher, most likely because haematological malignancies are more frequently recorded in the CPRD compared to cancer registries [23] and we used the new European standard population, published in 2013 [16], to calculate age-standardised incidence rates. We observed a trend of increasing CLL incidence rates with increasing year of study, although the difference did not reach statistical significance. Notably, between 2003 and 2009, annual incidence rates of all cancers in the UK also increased [22]. An observed decline in the incidence rate between 2011 and 2012 as shown in Fig. 2 was likely explained by the fact that data were only available through June 2012.

In our study, CLL occurred more often in males and elderly patients. More than a third of the CLL patients had comorbidities as defined by the Charlson comorbidity weighted index [17]. A similar pattern was seen in a Danish study of different types of cancer including leukaemia [24]. The average Charlson comorbidity weighted index in the CPRD population significantly decreased between 2000 and 2012. This is probably because high risk patients presenting with several comorbidities are being managed in the hospital and not in primary care. Although patients are currently diagnosed at an earlier disease stage [3], the median age at CLL diagnosis is still above 70 years and a substantial proportion of the patients have one or more comorbidities [5] as defined in the Charlson comorbidity weighted index [17]. The Cumulative Illness Rating Scale (CIRS), which is often used to assess the health status in patients with CLL, could not be applied $[25,26]$. The severity of morbidities recorded in the CPRD was not provided for all morbidities. For example, the CPRD coding system does not provide a code that allows a straightforward differentiation between mild, moderate or severe disease in the case of mental or metabolic illnesses.

During the time period from 2000 to 2012, average medical resource utilisation per year in terms of number of recorded referrals and hospitalisations increased significantly. As our trend analyses were controlled for duration of follow-up, the changes in medical resource utilisation may reflect real changes rather than a cohort effect. But the increase could also be due to changes in the way GPs recorded these referrals and hospitalisations. The OECD Health Data 2013 [27] found that the number of GP visits reported for the general UK population decreased over time. On the other hand, key statistics of the UK National Health Service (NHS) reported an increase in hospital admissions and outpatient appointments for the general UK population [28]. Changing clinical practice patterns may partly explain the change in medical resource utilisation. The decreasing number of referrals and hospitalisations found in the CPRD population in the second year compared to the first year following the CLL diagnosis could reflect referral back into the community for GP follow-up of low risk patients (i.e. to monitor disease progression). An increase of referrals and hospitalisations in the CPRD population could reflect need for immediate treatment after initial CLL diagnosis.

To our knowledge, this is the first study to describe population-based incidence rates and trends in medical resource utilisation in CLL patients using UK-based primary care data. Previous literature suggests that most of the diagnoses coded in the CPRD are accurately recorded and agree with other databases and national statistics [29] and cancer 
registries [23]. The validity of the CLL diagnosis was further assessed by screening random records of 100 CLL patients for CLL-concomitant laboratory measures, diagnostic tests and symptoms. The CLL diagnosis was considered validated if the patient record included at least one screening criterion for CLL. Only patients with at least 3 years of recorded history in the CPRD prior to the CLL diagnosis were included in our analysis to increase the probability of including only incident rather than prevalent cases Therefore, we are confident that the estimated incidence rates are reliable and generalizable to the UK population.

This study has several limitations that should be considered in interpreting our findings. Although about $70 \%$ of the CLL cases are diagnosed in asymptomatic patients [3] when they visit the GP for a routine blood count, some CLL cases may remain undetected because there is no visit to the GP for a routine blood count. Detection bias may limit the interpretation of our observed gender differences, as women may see the GP more often than men [30]. Total medical resource utilisation of CLL patients was incompletely assessed because details about secondary and tertiary care are not consistently recorded in the CPRD. Therefore, the recorded number of referrals and hospitalisations in the CPRD may be an underestimate. Details about type of chemotherapy regimen, for example, are often lacking as few chemotherapies are administered in the ambulatory setting.

Medical records of GPs are a valuable source to estimate population-based CLL incidences and trends in incidences in the UK. Further research to assess details of medical resource utilisation in secondary and tertiary care, especially regarding chemotherapy use, is needed to better understand total medical resource utilisation and treatment patterns in CLL.

\section{Conclusion}

Consistent with previous literature, we found that CLL incidence rates in the UK increased over time. However, in the past 5 years, we observed a stabilisation of CLL incidence rates in the CPRD population, which has not been previously reported in the literature. Further research to determine CLLspecific mortality and resource utilisation in non-primary care is needed as cancer patients are often treated in hospitals.

Acknowledgments We thank Pascal Egger for the technical support and programming of data extractions from the CPRD database.

\footnotetext{
Author contributions All authors were involved in the design of the study. A.M.P. was responsible for the first draft of the protocol, which was critically reviewed and approved by all authors. A.M.P was responsible for data analysis and the first draft of the manuscript. All authors contributed to data interpretation, critically reviewed all manuscript versions and read and agreed upon the final version of the manuscript.
}

Conflict of interest No financial support was received for the conduct of this study. All authors declare no conflicts of interest related to this article.

\section{References}

1. Rodriguez-Vicente AE, Diaz MG, Hernandez-Rivas JM (2013) Chronic lymphocytic leukemia: a clinical and molecular heterogenous disease. Cancer Genet 206(3):49-62. doi:10.1016/j.cancergen. 2013.01.003

2. Shanshal M, Haddad RY (2012) Chronic lymphocytic leukemia. Dis Mon 58(4):153-167. doi:10.1016/j.disamonth.2012.01.009

3. Montserrat E, Moreno C (2008) Chronic lymphocytic leukaemia: a short overview. Ann Oncol 19(Suppl 7):vii320-vii325. doi:10.1093/ annonc/mdn 460

4. Bhayat F, Das-Gupta E, Smith C, McKeever T, Hubbard R (2009) The incidence of and mortality from leukaemias in the UK: a general population-based study. BMC Cancer 9:252. doi:10.1186/14712407-9-252

5. Shanafelt T (2013) Treatment of older patients with chronic lymphocytic leukemia: key questions and current answers. Hematol Am Soc Hematol Educ Program 2013:158-167. doi:10.1182/asheducation2013.1.158

6. Hallek M (2013) Signaling the end of chronic lymphocytic leukemia: new frontline treatment strategies. Blood. doi:10.1182/blood-201305-498287

7. Hallek M, Fischer K, Fingerle-Rowson G, Fink AM, Busch R, Mayer J, Hensel M, Hopfinger G, Hess G, von Grunhagen U, Bergmann M, Catalano J, Zinzani PL, Caligaris-Cappio F, Seymour JF, Berrebi A, Jager U, Cazin B, Trneny M, Westermann A, Wendtner CM, Eichhorst BF, Staib P, Buhler A, Winkler D, Zenz T, Bottcher S, Ritgen M, Mendila M, Kneba M, Dohner H, Stilgenbauer S (2010) Addition of rituximab to fludarabine and cyclophosphamide in patients with chronic lymphocytic leukaemia: a randomised, openlabel, phase 3 trial. Lancet 376(9747):1164-1174. doi:10.1016/ S0140-6736(10)61381-5

8. Goede V, Fischer K, Busch R, Engelke A, Eichhorst B, Wendtner CM, Chagorova T, de la Serna J, Dilhuydy MS, Illmer T, Opat S, Owen CJ, Samoylova O, Kreuzer KA, Stilgenbauer S, Dohner H, Langerak AW, Ritgen M, Kneba M, Asikanius E, Humphrey K, Wenger M, Hallek M (2014) Obinutuzumab plus chlorambucil in patients with CLL and coexisting conditions. N Engl J Med 370(12): 1101-1110. doi:10.1056/NEJMoa1313984

9. Del Giudice I, Mauro FR, Foa R (2011) Chronic lymphocytic leukemia in less fit patients: "slow-go". Leuk Lymphoma 52(12):22072216. doi:10.3109/10428194.2011.606386

10. Lobetti-Bodoni C, Bertoni F, Stussi G, Cavalli F, Zucca E (2013) The changing paradigm of chronic lymphocytic leukemia management. Eur J Int Med 24(5):401-410. doi:10.1016/j.ejim.2013.03.009

11. Hallek M (2013) Chronic lymphocytic leukemia: 2013 update on diagnosis, risk stratification and treatment. Am J Hematol 88(9):803816. doi:10.1002/ajh.23491

12. Alexandropoulou K, van Vlymen J, Reid F, Poullis A, Kang JY (2013) Temporal trends of Barrett's oesophagus and gastrooesophageal reflux and related oesophageal cancer over a 10-year period in England and Wales and associated proton pump inhibitor and H2RA prescriptions: a GPRD study. Eur J Gastroenterol Hepatol 25(1):15-21. doi:10.1097/MEG.0b013e3283595086

13. Smiechowski B, Azoulay L, Yin H, Pollak MN, Suissa S (2013) The use of metformin and colorectal cancer incidence in patients with type II diabetes mellitus. Cancer Epidemiol Biomarkers Prev 22(10): 1877-1883. doi:10.1158/1055-9965.EPI-13-0196 
14. Jordan KP, Hayward RA, Blagojevic-Bucknall M, Croft P (2013) Incidence of prostate, breast, lung and colorectal cancer following new consultation for musculoskeletal pain: a cohort study among UK primary care patients. Int J Cancer 133(3):713-720. doi:10.1002/ijc.28055

15. Jick SS, Kaye JA, Vasilakis-Scaramozza C, Garcia Rodriguez LA, Ruigomez A, Meier CR, Schlienger RG, Black C, Jick H (2003) Validity of the general practice research database. Pharmacotherapy 23(5):686-689

16. Eurostat (2013) Revision of the European standard populationreport of Eurostat's task force. Methodologies \& working papers, vol 2013 edition. doi: $10.2785 / 11470$

17. Charlson ME, Pompei P, Ales KL, MacKenzie CR (1987) A new method of classifying prognostic comorbidity in longitudinal studies: development and validation. J Chron Dis 40(5):373-383

18. Al-Hashimi MM, Wang X (2013) Comparing the cancer in Ninawa during three periods (1980-1990, 1991-2000, 2001-2010) using Poisson regression. J Res Med Sci 18(12):1026-1039

19. Cox DR (1972) Regression models and life-tables. J R Stat Soc Ser B Methodol 34(2):187-220

20. Panovska A, Doubek M, Brychtova Y, Mayer J (2010) Chronic lymphocytic leukemia and focusing on epidemiology and management in everyday hematologic practice: recent data from the Czech Leukemia Study Group for Life (CELL). Clin Lymphoma Myeloma Leuk 10(4):297-300. doi:10.3816/CLML.2010.n.061

21. van den Broek EC, Kater AP, van de Schans SA, Karim-Kos HE, Janssen-Heijnen ML, Peters WG, Nooijen PT, Coebergh JW, Posthuma EF (2012) Chronic lymphocytic leukaemia in the
Netherlands: trends in incidence, treatment and survival, 19892008. Eur J Cancer 48(6):889-895. doi:10.1016/j.ejca.2011.06.053

22. Cancer Research U (2012) CancerStats - incidence 2009-UK

23. Boggon R, van Staa TP, Chapman M, Gallagher AM, Hammad TA, Richards MA (2013) Cancer recording and mortality in the General Practice Research Database and linked cancer registries. Pharmacoepidemiol Drug Saf 22(2):168-175. doi:10.1002/pds.3374

24. Coebergh JW, Janssen-Heijnen ML, Post PN, Razenberg PP (1999) Serious co-morbidity among unselected cancer patients newly diagnosed in the southeastern part of The Netherlands in 1993-1996. J Clin Epidemiol 52(12):1131-1136

25. Linn BS, Linn MW, Gurel L (1968) Cumulative illness rating scale. J Am Geriatr Soc 16(5):622-626

26. Miller MD, Paradis CF, Houck PR, Mazumdar S, Stack JA, Rifai AH, Mulsant B, Reynolds CF 3rd (1992) Rating chronic medical illness burden in geropsychiatric practice and research: application of the Cumulative Illness Rating Scale. Psychiatry Res 41(3):237-248

27. OECD (2013) OECD Health Data 2013. http://www.oecd.org/health/ health-systems/oecdhealthdata2013-frequentlyrequesteddata.htm

28. Confederation N (2013) Key statistics on the NHS. http://www. nhsconfed.org/PRIORITIES/POLITICAL-ENGAGEMENT/Pages/ NHS-statistics.aspx. Accessed 6 Jan 2014

29. Khan NF, Harrison SE, Rose PW (2010) Validity of diagnostic coding within the General Practice Research Database: a systematic review. Br J Gen Pract 60(572):e128-e136. doi:10.3399/bjgp10X483562

30. Santosh J, Crampton $P$ (2009) Gender differences in general practice utilisation in New Zealand. J Prim Health Care 1(4):261-269 